UBCTP 92-019

July 1992

\title{
Gauge theories on a cylinder
}

\author{
Edwin Langmann䍏 \\ and Gordon W. Semenofff \\ Department of Physics, The University of British Columbia \\ Vancouver, B.C., V6T 1Z1, Canada
}

\begin{abstract}
Gauge theory with massless fermions on a cylinder $(=(1+1)$ dimensional spacetime with compact space $S^{1}$ ) is studied in the Hamiltonian framework. Without using a gauge fixing condition, gauge degrees of freedom are eliminated by explicitly solving Gauss' law. The resulting effective Hamiltonian describes interacting fermions coupled to a finite number of quantum mechanical variables representing the physical Yang-Mills degrees of freedom. The method can be trivially extended to other gauge theories with matter on a cylinder.
\end{abstract}

\footnotetext{
${ }^{a}$ supported by the "Fonds zur Förderung der wissenschaftlichen Forschung" under the contract Nr. J0599-PHY

${ }^{b}$ on leave of absence of Institute of Theoretical Physics, TU-Graz, Austria

${ }^{c}$ supported in part by the Natural Sciences and Engineering Research Council of Canada.
} 
A rigorous construction and investigation of gauge theories with matter in $(3+1)$ dimensions is beyond present day's knowledge. $(1+1)$ dimensional models are much simpler and can be used as a testing ground to get more insight into gauge theories on a sound mathematical basis.

The identification of the physical degrees of freedom of non-Abelian gauge theories is highly non-trivial and crucial for understanding the physics of these models. Though trivial on a plane $(=$ spacetime $\mathbb{R} \times \mathbb{R})$, pure YM theory on a cylinder $\left(=\right.$ spacetime $\left.S^{1} \times \mathbb{R}\right)$ is non-trivial and has a finite number of physical degrees of freedom. This is most easily seen in the Hamiltonian framework: Here, the temporal component $A_{0}$ of the YM field is not dynamical but only a Lagrange multiplier enforcing Gauss' law (= invariance under all static gauge transformations), and the only gauge invariant quantities one can construct at some fixed time from the spatial component $A_{1}$ of the YM-field are the eigenvalues of the Wilson loop (holonomy) $W\left[A_{1}\right]$ over the whole space.

In a recent interesting paper Rajeev [1] presented a method to eliminate gauge degrees of freedom in pure YM theory on a cylinder and reduced it to a model for a free "particle" moving on a group manifold $\underline{G}$ (the structure group of the YM field) which can be solved exactly [2] ( $q=W\left[A_{1}\right] \in \underline{G}$ can be interpreted as position of the "particle" which due to invariance under rigid gauge transformations, $q \rightarrow h^{-1} q h \forall h \in \underline{G}$, effectively moves only on the Cartan subgroup of $\underline{G}$ [2]). The approach of [1] is very elegant as no gauge 
fixing condition is imposed but Gauss' law is explicitly solved resulting in an effective Hamiltonian $\mathbf{H}_{\text {eff }}$ on the physical Hilbert space $\mathcal{H}_{\text {phys }}$ of the model.

In this Letter we extend the method of Rajeev [1] to massless fermions coupled to a Yang-Mills field with gauge group $\underline{G}=\mathrm{SU}(N)$ or $\mathrm{U}(N)$ on a cylinder. The construction is done on a semiclassical level with the fermion field algebra in the naive (unphysical) representation (no filled Dirac sea) as this simplifies the arguments and allows for a straightforward extension to other gauge theories with matter on a cylinder. We note that a rigorous construction of this model and the elimination of the gauge degrees of freedom can be done also on the full quantum level, and that the final result does not depend on whether one goes to the full quantum level before or after this elimination (we hope to report on that in a future publication).

We start with the Lagrangian density'

$$
\mathcal{L}=\bar{\psi} \gamma^{\nu}\left(-\mathrm{i} \partial_{\nu}+A_{\nu}\right) \psi-\frac{1}{2 e^{2}} \operatorname{tr}\left(F_{\nu \mu} F^{\nu \mu}\right)
$$

with? $A_{\nu} \equiv A_{\nu}^{a} T^{a}$ the YM field, $F_{\nu \mu} \equiv \partial_{\nu} A_{\mu}-\partial_{\mu} A_{\nu}+\mathrm{i}\left[A_{\nu}, A_{\mu}\right]$ with $\nu, \mu \in$ $\{0,1\}$ spacetime indices, $\psi, \bar{\psi} \equiv \psi^{*} \gamma^{0}$ the fermion fields, and $e$ the coupling constant; more explicitly, $\psi^{(*)}(x) \equiv \psi_{\sigma, \mathrm{A}}^{(*)}(x), T^{a} \equiv T_{\mathrm{AB}}^{a}, \gamma^{\nu} \equiv \gamma_{\sigma \sigma^{\prime}}^{\nu}$ with $\sigma, \sigma^{\prime} \in\{1,2\}$ and $\mathrm{A}, \mathrm{B} \in\{1,2 \ldots N\}$ the spin and color indices, respectively.

\footnotetext{
${ }^{1} \partial_{\nu} \equiv \partial / \partial x^{\nu}$ with $x^{0} \equiv t$ (time), $x^{1} \equiv x \in[0,2 \pi)$ (spatial coordinate); our metric tensor is $g_{\nu \mu}=\operatorname{diag}(1,-1)$

${ }^{2}$ the $T^{a}=\left(T^{a}\right)^{*}$ are the generators of the Lie algebra $\underline{g}$ of $\underline{G}$ in the fundamental representation normalized to that $\operatorname{tr}\left(T^{a} T^{b}\right)=\delta^{a b} / 2(\operatorname{tr}(\cdot)$ is the $N \times N$-matrix trace)
} 
By the usual canonical procedure [3] we obtain the Hamiltonian (we assume periodic boundary conditions for all fields)

$$
\mathbf{H}=\int_{0}^{2 \pi} \mathrm{d} x\left(\operatorname{tr}\left(e^{2} \Pi_{1}(x)^{2}-2 A_{0}(x) G(x)\right)+\psi^{*}(x) \gamma_{5}\left(-\mathrm{i} \partial_{1}+A_{1}(x)\right) \psi(x)\right)
$$

where $\Pi_{\nu}^{a}(x)=F_{0 \nu}^{a}(x) / e^{2}$ and $\mathrm{i} \psi_{\sigma, \mathrm{A}}^{*}(x)$ are the canonical momenta to $A^{a, \nu}(x)$ and $\psi_{\sigma, \mathrm{A}}(x)$, respectively, $\gamma_{5}=-\gamma^{0} \gamma^{1}$, and 5 .

$$
G(x) \equiv-\partial_{1} \Pi_{1}(x)-\mathrm{i}:\left[A_{1}(x), \Pi_{1}(x)\right]:+\rho(x)
$$

with $\rho^{a}(x)=\psi^{*}(x) T^{a} \psi(x)$; the normal ordering : $\cdots:$ means that terms coming from the non-commutativity of $A_{1}^{a}(x)$ and $\Pi_{1}^{b}(y)$ have to be discarded. Moreover, we have the following canonical (anti-) commutator relations

$$
\begin{array}{r}
{\left[A_{\nu}^{a}(x), \Pi_{\mu}^{b}(y)\right]=-\mathrm{i} g_{\nu \mu} \delta^{a b} \delta(x-y)} \\
\left\{\psi_{\sigma, \mathrm{A}}(x), \psi_{\sigma^{\prime}, \mathrm{B}}^{*}(y)\right\}=\delta_{\sigma \sigma^{\prime}} \delta_{\mathrm{AB}} \delta(x-y)
\end{array}
$$

with the other (anti-) commutators vanishing as usual. From the primary constraint $\Pi_{0}(x) \simeq 0$ we get the secondary constraint $G(x) \simeq 0$ (Gauss' law). Following Rajeev [1] we introduce the Wilson line $S(x) \equiv S_{\mathrm{AB}}(x)$ as the (unique) solution of

$$
\left(\partial_{1}+\mathrm{i} A_{1}(x)\right) S(x)=0, \quad S(0)=1
$$

\footnotetext{
${ }^{3}$ we use the notation $G(x) \equiv G^{a}(x) T^{a}$ and similarly for $A_{\nu}, \Pi_{\nu}, \rho$ etc.

$4[\cdot, \cdot]$ is the commutator and $\{\cdot, \cdot\}$ the anticommutator
} 
This allows us to write Gauss' law as

$$
\tilde{G}(x)=-\partial_{1} \tilde{\Pi}_{1}(x)+\tilde{\rho}(x) \simeq 0
$$

with $\tilde{G}(x) \equiv: S(x)^{-1} G(x) S(x)$ : and similarly for $\Pi_{1}$ and $\rho$. In this form, Gauss' law is easily solved: $\tilde{\Pi}_{1}(x) \simeq \Pi_{1}(0)+R(x)$ with $R(x)=\int_{0}^{x} \mathrm{~d} y \tilde{\rho}(y)$. Introducing $p \equiv \int_{0}^{2 \pi} \mathrm{d} x \tilde{\Pi}_{1}(x) / 2 \pi$ we obtain

$$
\tilde{\Pi}_{1}(x) \simeq p+\bar{R}(x), \quad \bar{R}(x)=R(x)-\int_{0}^{2 \pi} \frac{\mathrm{d} y}{2 \pi} R(x),
$$

and from $\Pi_{1}(0)=\Pi_{1}(2 \pi)$ we get the constraint

$$
p+\bar{R}(2 \pi)=: q^{-1}(p+\bar{R}(0)) q:
$$

with $q \equiv S(2 \pi)$ identical with the Wilson loop $W\left[A_{1}\right]$. Due to the cyclicity of the trace, we obtain from (2) the effective Hamiltonian

$$
\begin{array}{r}
\mathbf{H}_{\text {eff }}^{\prime}=\int_{0}^{2 \pi} \mathrm{d} x\left(\operatorname{tr}\left(e^{2} \tilde{\Pi}_{1}(x)^{2}\right)+\tilde{\psi}^{*}(x) \gamma_{5}\left(-\mathrm{i} \partial_{1}\right) \tilde{\psi}(x)\right) \\
=e^{2} 2 \pi \operatorname{tr}\left(p^{2}\right)+\int_{0}^{2 \pi} \mathrm{d} x\left(\operatorname{tr}\left(e^{2} \bar{R}(x)^{2}\right)+\tilde{\psi}^{*}(x) \gamma_{5}\left(-\mathrm{i} \partial_{1}\right) \tilde{\psi}(x)\right)
\end{array}
$$

with $\tilde{\psi}(x) \equiv S(x)^{-1} \psi(x)$. As $\tilde{\rho}^{a}(x)=2 \operatorname{tr}\left(T^{a} S(x)^{-1} T^{b} S(x)\right) \psi^{*}(x) T^{b} \psi(x)$, it follows from the invariance of $T^{a} \otimes T^{a}$,

$$
T_{A B}^{a} T_{C D}^{a}=\left(h^{-1} T^{a} h\right)_{A B}\left(h^{-1} T^{a} h\right)_{C D} \quad \forall h \in \underline{G},
$$

that

$$
\tilde{\rho}^{a}(x)=\tilde{\psi}^{*}(x) T^{a} \tilde{\psi}(x) .
$$


Note that the fermion fields $\tilde{\psi}^{(*)}$ still obey the canonical anticommutator relations, but they have twisted boundary conditions

$$
\tilde{\psi}(2 \pi)=q^{-1} \tilde{\psi}(0)
$$

It is easy to see that the Wilson loop variables obey

$$
\left[p^{a}, q\right]=-q T^{a}, \quad\left[p^{a}, q^{-1}\right]=T^{a} q^{-1}
$$

The canonical anticommutator relations imply $\left[\bar{R}^{a}(0), \tilde{\psi}^{*}(x)\right]=\left(\frac{x}{2 \pi}-1\right) \tilde{\psi}^{*}(x) T^{a}$, and with $p^{a}=\Pi_{1}^{a}(0)-\bar{R}^{a}(0)$ we obtain

$$
\left[p^{a}, \tilde{\psi}^{*}(x)\right]=-\frac{x}{2 \pi} \tilde{\psi}^{*}(x) T^{a}
$$

At this stage we have formulated the model entirely in terms of the Wilson loop variables $p=p^{a} T^{a}, q$, and the twisted fermion fields $\tilde{\psi}^{(*)}$ obeying the relations given above. $\mathbf{H}_{\text {eff }}^{\prime}$ has to be regarded as an operator on the tensor product $\mathcal{H}_{\text {phys }}^{\prime}$ of the Hilbert space of the Wilson loop variables [2] and an appropriate fermion Fock space.

$\mathcal{H}_{\text {phys }}^{\prime}$ is not quite the physical Hilbert space of the model as we still have the constraint (8) which is associated with the invariance under rigid gauge transformations

$$
\begin{aligned}
q & \rightarrow h^{-1} q h \\
p & \rightarrow h^{-1} p h \\
\tilde{\psi}(x) & \rightarrow h^{-1} \tilde{\psi}(x) \quad \forall h \in \underline{G} .
\end{aligned}
$$


Indeed, it is easy to check that eqs. (8), (9), (11), (12), and (13) are invariant under (14).

To eliminate this constraint, it is convenient to introduce an algebraic basis in the Lie algebra $\underline{g}$ of $\underline{G}$ as follows. Let $e_{i j}$ be the $N \times N$ matrix with the elements $\left(e_{i j}\right)_{k l}=\delta_{i k} \delta_{j l}$. We define $H_{i}=e_{i, i}-e_{i+1, i+1}$ for $i=1, \ldots, N-1$, $E_{1}^{+}=e_{1,2}, E_{2}^{+}=e_{1,3}, \ldots, E_{N-1}^{+}=e_{1, N}, E_{N}^{+}=e_{2,3}, \ldots, E_{\frac{1}{2} N(N-1)}^{+}=e_{N-1, N}$, and $E_{j}^{-}=\left(E_{j}^{+}\right)^{*}$. These matrices obey

$$
\begin{aligned}
{\left[H_{i}, H_{j}\right] } & =0 \\
{\left[H_{i}, E_{j}^{ \pm}\right] } & = \pm a_{i j} E_{j}^{ \pm} \quad \forall i, j
\end{aligned}
$$

with $a_{i j}$ the elements of $(N-1) \times \frac{1}{2} N(N-1)$ matrix given by $a_{i j}=\delta_{i k(j)}-$ $\delta_{i l(j)}-\delta_{i+1, k(j)}+\delta_{i+1, l(j)}$ where $k(j), l(j)$ are determined from $E_{j}^{+}=e_{k(j), l(j)}$. Moreover,

$$
\begin{array}{cl}
H_{i}^{*}=H_{i}, & \left(E_{i}^{+}\right)^{*}=E_{i}^{-}, \\
\operatorname{tr}\left(H_{i} H_{j}\right)=b_{i j} \quad \operatorname{tr}\left(E_{i}^{+} E_{j}^{-}\right)=\delta_{i j}, & \\
\operatorname{tr}\left(H_{i} E_{j}^{ \pm}\right)=\operatorname{tr}\left(E_{i}^{ \pm} E_{j}^{ \pm}\right)=0 \quad \forall i, j
\end{array}
$$

where $b$ is the $(N-1) \times(N-1)$ matrix with elements $b_{i j}=2 \delta_{i j}-\delta_{i+1, j}-\delta_{i, j+1}$. Note that $b$ is invertible. Obviously the matrices $H_{i}, i=1, \ldots, N-1$ and $E_{j}^{ \pm}$, $j=1, \ldots, \frac{1}{2} N(N-1)$, span the Lie algebra of $\underline{G}=\mathrm{SU}(N)$. For $\underline{G}=\mathrm{U}(N)$ we have to add to these $H_{0}=1$ (the $N \times N$ unit matrix) and set $a_{0 j}=0$,

${ }^{5}$ by induction one can show that its determinant is $N \neq 0$ 
$b_{0 j}=b_{j 0}=N \delta_{j 0}$ so that the relations above still hold. Then we can write

$$
X=\sum_{j}\left(X^{0, j} H_{j}+X^{+, j} E_{j}^{-}+X^{-, j} E_{j}^{+}\right) \quad \forall X \in \underline{g}
$$

with $X^{0, j}=\sum_{k}\left(b^{-1}\right)^{j k} \operatorname{tr}\left(H_{k} X\right)=\left(X^{0, j}\right)^{*}$ with $\left(b^{-1}\right)^{j k}$ the elements of the inverse matrix of $b$, and $X^{ \pm, j}=\operatorname{tr}\left(E_{j}^{ \pm} X\right)=\left(X^{\mp, j}\right)^{*}$.

Every $q \in \underline{G}$ can be represented as $q=h_{q} d h_{q}^{-1}$ with $d$ in the Cartan subgroup of $\underline{G}, d=\exp \left(-2 \pi \mathrm{i} \sum_{i} Y^{i} H_{i}\right)$ (note that this representation is not unique). We can explicitly solve the constraint (8) by performing a rigid gauge transformation (14) with $h=h_{q}$. Denoting for simplicity the gauge transformed $q, \tilde{\psi}$ etc. by the same symbol, we can represent $\tilde{\psi}$ as

$$
\tilde{\psi}(x) \simeq d^{-x / 2 \pi} \psi(x)
$$

with $\psi(x)$ free fermion fields obeying periodic boundary conditions. Performing a Fourier transform, $\psi(x)=\sum_{n \in \mathbb{Z}} \hat{\psi}(n) \exp (\mathrm{i} n x) / \sqrt{2 \pi}$, and using

$$
\begin{aligned}
\exp \left(-\mathrm{i} \sum_{i} \alpha^{i} H_{i}\right) H_{j} \exp \left(\mathrm{i} \sum_{i} \alpha^{i} H_{i}\right) & =H_{j} \\
\exp \left(-\mathrm{i} \sum_{i} \alpha^{i} H_{i}\right) E_{j}^{ \pm} \exp \left(\mathrm{i} \sum_{i} \alpha^{i} H_{i}\right) & =E_{j}^{ \pm} \exp \left(\mp \mathrm{i} \sum_{i} \alpha^{i} a_{i j}\right)
\end{aligned}
$$

for all real $\alpha^{i}$ following from (15), we obtain from (8) by a straightforward but tedious calculation that $\hat{\rho}^{0, j}(0) \simeq 0$ and

$$
\left(1-\mathrm{e}^{\mp 2 \pi \mathrm{i} \sum_{i} Y^{i} a_{i j}}\right)\left(p^{ \pm, j}+\frac{1}{4 \pi^{2}} \sum_{n \in \mathbb{Z}} \frac{\hat{\rho}^{ \pm, j}(n)\left(\mathrm{e}^{\mp 2 \pi \mathrm{i} \sum_{i} Y^{i} a_{i j}}-1\right)}{\left(n \mp \sum_{i} Y^{i} a_{i j}\right)^{2}}\right) \simeq 0
$$

where $\hat{\rho}(n)=\int_{0}^{2 \pi} \mathrm{d} x \rho(x) \exp (-\mathrm{i} n x)$, and with that

$$
\tilde{\Pi}(x) \simeq p+\bar{R}(x) \simeq \sum_{j} p^{0, j} H_{j}+\frac{1}{2 \pi} \sum_{n \in \mathbb{Z}} \sum_{j}\left(\frac{\hat{\rho}^{0, j}(n)}{\mathrm{i} n} \mathrm{e}^{\mathrm{i} n x} H_{j}\left(1-\delta_{n 0}\right)\right.
$$




$$
\left.+\frac{\hat{\rho}^{+, j}(n)}{\mathrm{i}\left(n-\sum_{i} Y^{i} a_{i j}\right)} \mathrm{e}^{\mathrm{i}\left(n-\sum_{i} Y^{i} a_{i j}\right) x} E_{j}^{-}+\frac{\hat{\rho}^{-, j}(n)}{\mathrm{i}\left(n+\sum_{i} Y^{i} a_{i j}\right)} \mathrm{e}^{\mathrm{i}\left(n+\sum_{i} Y^{i} a_{i j}\right) x} E_{j}^{+}\right) .
$$

From (12) we get $\left[p^{0, j}, d\right]=-d \frac{1}{2} \sum_{k}\left(b^{-1}\right)^{j k} H_{k}$. . Hence we can represent the $p^{0, j}$ as $\frac{1}{4 \pi \mathrm{i}} \sum_{k}\left(b^{-1}\right)^{j k} \frac{\partial}{\partial Y^{k}}$. Putting this all together, we obtain from (9)

$$
\begin{array}{r}
\mathbf{H}_{\mathrm{eff}}=-\frac{e^{2}}{8 \pi} \sum_{j} \frac{\partial^{2}}{\partial Y_{j} \partial Y^{j}}+\sum_{n \in \mathbb{Z}} \hat{\psi}^{*}(n) \gamma_{5}\left(n+\sum_{j} Y^{j} H_{j}\right) \hat{\psi}(n)+ \\
\frac{e^{2}}{2 \pi} \sum_{n \in \mathbb{Z}} \sum_{j}\left(\left(1-\delta_{0 n}\right) \frac{\hat{\rho}^{0, j}(n) \hat{\rho}_{j}^{0}(-n)}{n^{2}}+\frac{\hat{\rho}^{+, j}(n) \hat{\rho}^{-, j}(-n)}{\left(n-\sum_{i} Y^{i} a_{i j}\right)^{2}}+\frac{\hat{\rho}^{-, j}(n) \hat{\rho}^{+, j}(-n)}{\left(n+\sum_{i} Y^{i} a_{i j}\right)^{2}}\right)
\end{array}
$$

with $\frac{\partial}{\partial Y_{j}} \equiv \sum_{k}\left(b^{-1}\right)^{j k} \frac{\partial}{\partial Y^{k}}$ and $\hat{\rho}_{j}^{0} \equiv \sum_{k} b_{j k} \hat{\rho}^{0, k}$. This is our final result.

It should be pointed out that (18) is identical with the Hamiltonian one obtains by fixing the gauge in eqs. (2), (3) to

$$
A_{0}(x)=0, \quad A_{1}(x)=\sum_{j} Y^{j} H_{j}
$$

which is essentially the Coulomb gauge. Thus the construction above can be regarded as an justification of that gauge.

It is important to note that though we have eliminated in eq. (18) as many gauge degrees of freedom as possible, there are still gauge transformations left, namely all those that leave the diagonalized Wilson loop $d$ in the Cartan subgroup of $\underline{G}$. For example, $h(x)=\exp \left(\mathrm{i} x \sum_{j} \nu^{j} H_{j}\right)$ with $\nu^{j} \in \mathbb{Z}$ is a gauge transformation (i.e. $h(0)=h(2 \pi)=1$ ) leaving $d$ invariant but acting nontrivially on the $Y^{j}: Y^{j} \rightarrow Y^{j}+\nu^{j}$ (it is straightforward to check that (18) is invariant under these gauge transformations). This can be understood as

\footnotetext{
${ }^{6}$ we used $T_{\mathrm{AB}}^{a} T_{\mathrm{CD}}^{a}=\frac{1}{2} \delta_{\mathrm{AD}} \delta_{\mathrm{BC}}-\alpha \delta_{\mathrm{AB}} \delta_{\mathrm{CD}}$ with $\alpha=\frac{1}{2 N}$ for $\underline{G}=\mathrm{SU}(N)$ and 0 for $\underline{G}=\mathrm{U}(N)$
} 
Gribov ambiguity [4]: the Coulomb gauge condition $\partial A_{1}(x) / \partial x=0$ does not uniquely determine one representative in each gauge orbit. Demanding that the physical states of the model are invariant also under these discrete gauge transformations leads to a highly non-trivial vacuum structure on the full quantum level similar to the one of the Schwinger model [5]. We intent to report on that in a future publication.

\section{References}

[1] Rajeev S. G., Yang-Mills theory on a cylinder, Phys. Lett. 212B, 203205 (1988).

[2] Dowker J. S., Quantum mechanics on group space and Huygens' principle, Ann. Phys. 62, 361 (1971).

[3] Sundermeyer K., Constrained Dynamics, Lecture Notes in Physics 169, Springer Verlag (1982).

[4] Gribov V. N., Quantization of non-Abelian gauge theories, Nucl.Phys. B 139, 1 (1978).

[5] Manton N. S., The Schwinger model and its axial anomaly, Ann. Phys. 159, 220-251 (1985). 